\title{
Clinical outcomes and second-look arthroscopic findings of anterior cruciate ligament reconstruction with autograft, hybrid graft, and allograft
}

Xiaozuo Zheng ${ }^{1,2^{*}}$ (D), Yang $\mathrm{Hu}^{3}$, Peng Xie ${ }^{2,4}$, Tong $\mathrm{Li}^{1,2}$, Yu-e Feng ${ }^{1,2}$, Juyuan $\mathrm{Gu}^{1,2}$ and Shijun Gao ${ }^{1,2^{*}}$

\begin{abstract}
Background: There is no consensus as to the choice of grafts for primary anterior cruciate ligament (ACL) reconstruction. The purpose of this study was to compare the clinical and second-look arthroscopic outcomes after ACL reconstruction by use of autograft, hybrid graft, and $y$-irradiated allograft.

Methods: Ninety-seven patients who underwent second-look arthroscopy after ACL reconstruction with autografts (28 patients, hamstring autograft), hybrid grafts (32 patients, hamstring autograft augmented with $y$-irradiated tibialis anterior tendon allograft), or $\gamma$-irradiated allografts (37 patients, tibialis anterior tendons) were included in this study. The clinical outcomes were compared by using Lysholm score, International Knee Documentation Committee (IKDC) score, and Tegner activity score, and the side-to-side differences of KT-1000 measurement. Second-look arthroscopic findings were compared in terms of synovial coverage and graft tension.

Results: There were no statistical significances among the three groups in Lysholm score, IKDC score, or Tegner activity score $(P>0.05)$. The KT-1000 examination showed more anterior laxity in the $\gamma$-irradiated allograft group than in the autograft or hybrid graft groups ( $P=0.006$, and $P=0.013$, respectively). Two patients in the autograft group, 2 patients in the hybrid graft group and 4 patients in the allograft group were evaluated as graft failure on second-look arthroscopy. The synovial coverage was superior in the autograft group than that in the hybrid graft group or the allograft group $(P=0.013$ and $P=0.010$, respectively), and was comparable between the hybrid graft group and allograft group $(P=0.876)$. With regard to graft tension, the autograft group and hybrid group were comparable ( $P=0.883$ ) but showed better results than the allograft group $(P=0.011$ and $P=0.007$, respectively).

Conclusion: The hamstring autografts and hybrid grafts used for $A C L$ reconstruction produced equal efficacy but provided better knee stability than allografts. In addition, the hamstring autografts showed better synovial coverage than the other two graft types.
\end{abstract}

Keywords: Anterior cruciate ligament, Reconstruction, Autograft, Hybrid, Allograft, Second-look

\footnotetext{
*Correspondence: xiaozuo_zheng@163.com; 13595933@qq.com

1 Department of Orthopedics, Third Hospital of Hebei Medical University, No.

139 Ziqiang Road, Shijiazhuang 050051, China

Full list of author information is available at the end of the article
}

(c) The Author(s). 2019 Open Access This article is distributed under the terms of the Creative Commons Attribution 4.0 International License (http://creativecommons.org/licenses/by/4.0/), which permits unrestricted use, distribution, and reproduction in any medium, provided you give appropriate credit to the original author(s) and the source, provide a link to the Creative Commons license, and indicate if changes were made. The Creative Commons Public Domain Dedication waiver (http://creativecommons.org/publicdomain/zero/1.0/) applies to the data made available in this article, unless otherwise stated. 


\section{Background}

Anterior cruciate ligament (ACL) reconstruction is currently regarded as the best treatment for physically active patients with ACL rupture. A variety of autograft, hybrid graft, and allograft tissues are used for primary ACL reconstruction. Hamstring autograft is a popular choice due to the advantages of low donor site morbidity, early graft incorporation, and no risks of immune reactions and disease transmission [1, 2]. However, some patients may have small tendon diameters, which compromises the tensile strength of the grafts [3]. Clinically, this has translated to a higher likelihood of poor clinical outcomes as the graft diameter decreases. Previous studies have reported that the use of hamstring autografts with $8 \mathrm{~mm}$ in diameter or less resulted in increased graft failure risk and anterior knee laxity [4-6]. Allografts have been shown to be a reasonable alternative to small hamstring autografts. The major advantage of using allograft for ACL reconstruction is the availability of desired graft size with no donor site morbidities [7]. Some controlled clinical studies have reported comparable results with soft-tissue allografts and hamstring tendon autografts $[8,9]$, whereas others reported that the allograft tendons might have inferior graft maturity, higher risk of graft failure, and increased knee laxity than autograft tendons in ACL reconstruction $[2,10,11]$. Another solution to an inadequate graft diameter is hybrid graft which comprises autograft hamstrings and allograft soft tissues. It enables surgeons to customize graft size without harvesting additional autograft. However, only a few studies compared the clinical outcomes between hamstring autografts and hybrid grafts used for ACL reconstruction, and whether allograft augmented hamstrings are effective is still debatable. Leo et al. [12] and Li et al. [10] reported that the use of a hybrid graft has a comparable retear rate, knee stability, and patient-reported scores compared with the use of autograft hamstring. Jacobs et al. reported that allograft augmentation of hamstring autograft reduced the revision risk for young patients undergoing ACL reconstruction [13]. By contrast, other studies showed opposite results where inferior outcomes were observed for hybrid grafts compared with those of autografts [14-16]. Therefore, it is still unclear which of the three types of grafts, including hamstring autograft, hybrid graft, and soft-tissue allograft, is the optimal option for primary ACL reconstruction.

All biological tissues, whether allograft or autograft, undergo a similar ligamentization process when implanted as an ACL substitute [17]. Previous studies have reported that the quality of graft ligamentization has strong correlation with long-term survival of the grafts $[18,19]$. Second-look arthroscopy is a less invasive method for evaluating the graft integrity and remodeling process of the reconstructed ACL [20-22]. Although some studies have reported second-look arthroscopic findings after ACL reconstruction, there is a paucity of research on the comparison of clinical outcome differences and graft morphology among different graft types.

The purpose of this study was to compare the patient-reported outcomes, knee stability, and secondlook arthroscopic findings of patients who underwent ACL reconstruction with hamstring autograft, hybrid graft, or soft-tissue allograft. We hypothesized that the outcomes of ACL reconstruction with hamstring tendon autograft would have better results compared with those with hybrid grafts or allografts, and similar outcomes would be seen in the patients with hybrid grafts and allografts.

\section{Methods}

\section{Participants}

This study was carried out with the approval of the ethics committee of our institution. Patients who underwent second-look arthroscopy after anatomic single-bundle ACL reconstruction were retrospectively reviewed, and signed informed consent was obtained from each participant. The inclusion criteria were (1) primary ACL tears with single-bundle reconstruction; (2) the grafts with diameters equal to or more than $8 \mathrm{~mm}$ including hamstring autografts (combined gracilis and semitendinosus tendons), hybrid grafts (combined hamstring autograft and $\gamma$ irradiated tibialis anterior allograft), or $\gamma$-irradiated tibialis anterior allografts; (3) unilateral knee with no history of previous surgery; (4) skeletally mature patients aged more than 16 years old; (5) no signs of cartilage lesion and osteoarthritis; and (6) no combined ligament injury that requires surgical intervention. The exclusion criteria were (1) revision ACL reconstruction, (2) multiple knee ligaments requiring surgical intervention, (3) serious meniscal tear requiring total meniscectomy, and (4) patients who were unable to comply with the treatment protocol, or could not finish at least 12 months follow-up.

\section{Grafts preparation and surgical ACL reconstruction}

For the autograft reconstruction, the semitendinosus and gracilis tendons were harvested and prepared as a 4stranded or 6-stranded hamstring autograft. If the combined diameter of the autograft tendons was less than 8 $\mathrm{mm}$, the $\gamma$-irradiated tibialis anterior allograft was used as augmentation to achieve a minimum desired diameter of $8 \mathrm{~mm}$. In the allograft group, the $\gamma$-irradiated tibialis anterior allograft was prepared as a 2-stranded or 4stranded graft. All the allografts were irradiated at a dose of $2.5 \mathrm{Mrad}$, and supplied by a certified tissue bank (Shanxi OsteoRad Biomateral Co., Ltd., Taiyuan, China).

Diagnostic arthroscopy was performed to identify the ACL tear. The combined meniscal injuries were addressed as needed before ACL reconstruction. The ACL 
remnant was generally preserved. All patients underwent anatomic single-bundle ACL reconstruction, with the femoral and tibial tunnel placed in the center of the femoral and tibial ACL insertion sites. The tunnel diameter was equal to the graft tendon diameter. The graft was then pulled into both tunnels from tibia to femur. The femoral side was fixed with a TightRope device (Arthrex, Naples, FL, USA), and the tibial side was fixed with an interference screw (Arthrex, Naples, FL, USA).

\section{Rehabilitation protocol}

All patients followed the same rehabilitation protocol. The knee was immobilized with a long hinge brace immediately postoperatively for up to 8 weeks. Isometric quadriceps training was started immediately after surgery. Range-of-motion (ROM) exercise was started 2 days after the operation, with the goal of gaining normal ROM within 6 weeks. Partial weight bearing was allowed in the first 4 weeks, and full weight bearing was started at 8 weeks. Patients were allowed to run after 6 months, and return to sports activities gradually at 9 months after surgery. The patients were routinely followed up at 1,3 , 6, 9, 12 and 24 months, and clinical outcomes and second-look arthroscopic evaluation were recorded at the final follow-up postoperatively.

\section{Clinical evaluation}

Subjective functional assessment included Lysholm score, subjective International Knee Documentation Committee (IKDC) score, and Tegner activity score. Knee stability was evaluated by the side-to-side differences of KT-1000 arthrometer measurement at 30-lb. force (MEDmetric, San Diego, CA, USA). The complications due to surgery were also recorded.

\section{Second-look arthroscopic evaluation}

Second-look arthroscopy was performed only in patients who requested femoral fixation device removal at least 12 months after ACL reconstruction. The purpose and risk of second-look arthroscopy were explained, and the informed consents were provided by all the patients. Graft failure was defined as a failure in the completion of the ligamentization process, leading to an atonic, disorganized, and non-viable graft [23] (Fig. 1). Graft healing in relation to synovial coverage, and graft thickness and tension were evaluated. Synovial coverage of the grafts was graded as "good" (completely or more than $80 \%$ covered), "fair" (50-80\% covered), or "poor" (less than $50 \%$ or barely covered) [20, 24] (Fig. 2). Graft tension was evaluated using a probe with the knee in positions from extension to flexion. The middle part of the graft was manually measured and graded as "taut" (as tense as normal ACL), mildly lax (less tense than the

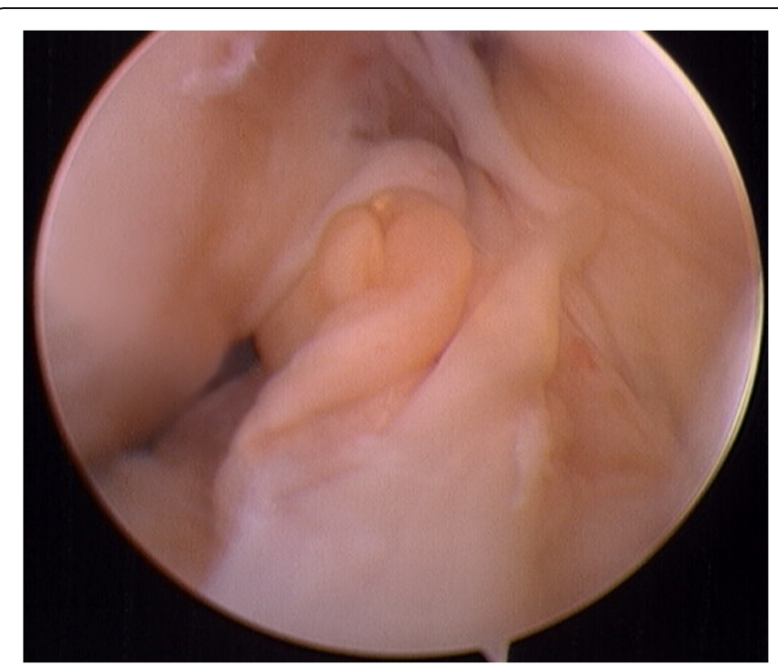

Fig. 1 Biological failure defined as an atonic, disorganized, and nonviable ACL graft under second-look arthroscopy

normal ACL), and lax (complete tear or obvious loss of tension) $[24,25]$.

\section{Statistical analysis}

The baseline characteristics of the three groups were compared using chi-square test for nominal variables, and analysis of variance (ANOVA) and least significant difference (LSD) test were conducted for continuous variables. The Kruskal-Wallis test and Mann-Whitney $U$ test were used to test differences of the synovial coverage and graft tension among the three groups. Comparison of the knee functional scores and differences in KT-1000 were analyzed by ANOVA and LSD test. The level of significance was set at $P<0.05$. SPSS software (version 19.0; SPSS Inc., Chicago, Illinois) was used for data analysis.

\section{Results}

\section{Patient demographics}

From January 2013 to February 2018, 97 patients who met the inclusion criteria and underwent second-look arthroscopy were enrolled, including 28 with autograft ACL reconstruction, 32 with hybrid graft ACL reconstruction, and 37 with allograft ACL reconstruction. There was no statistically significant difference in demographics at the time of initial ACL reconstruction among the groups (Table 1). The mean follow-up period of all the patients was $20.1 \pm 6.7$ months with no significant difference among the groups. The graft diameter was significantly larger in the hybrid group than the autograft and allograft groups $(P=0.000$ and 0.012 , respectively), but there was no significant difference between the autograft and allograft groups $(P=0.086)$. 


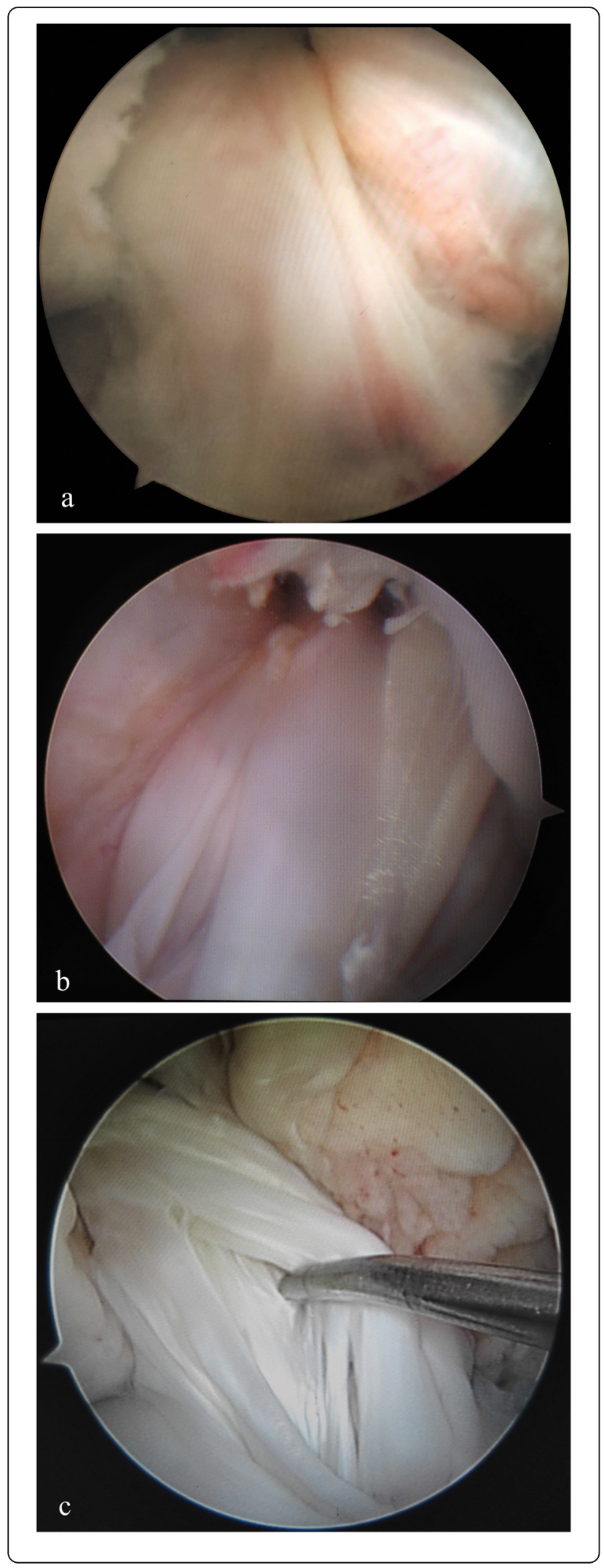

Fig. 2 Arthroscopic classification of the reconstructed $A C L$ graft based on synovial coverage. a Completely or more than $80 \%$ synovial coverage over the graft. b $50-80 \%$ of the synovial coverage over the graft. c Less than $50 \%$ or barely synovial coverage over the graft

\section{Complications}

There was no case of deep venous thrombosis, neurovascular injury, deep infection, and fixation failure in any group. One patient with wound disunion at the tibial incision area underwent debridement in the hybrid graft group. One patient with serious ROM deficit due to arthrofibrosis underwent surgical arthrolysis in the autograft group.

\section{Knee function and stability}

No significant difference was found with respect to Lysholm score, subjective IKDC score, or Tegner activity score among the 3 groups at final follow-up assessments $(P>0.05)$ (Table 2).

The KT-1000 side-to-side differences at final followup were significantly inferior in allograft group than those in the autograft group and hybrid group $(P=0.006$ and 0.013 , respectively). Additionally, no significant difference was found between the autograft group and hybrid group $(P=0.748)$ (Table 3$)$.

\section{Second-look arthroscopy}

Two patients in the autograft group, 2 patients in the hybrid graft group, and 4 patients in allograft group were evaluated as graft failure on second-look arthroscopy. There were 1 patient with failed autograft, 1 patient with failed hybrid grafts, and 3 patients with failed allografts, and they underwent revision ACL reconstruction. The other patients did not received revision surgery because there was no feeling of instability during daily activities. With respect to synovial coverage over the graft, the autograft group showed significantly better results than the other two groups $(P=0.016$ among the 3 groups, $P=0.013$ for autograft group vs. hybrid graft group, $P=0.010$ for autograft group vs. allograft group); however, there was no significant difference between hybrid graft group and allograft group $(P=0.876)$. With respect to graft tension, both the autograft and hybrid graft groups showed statistically significant differences compared with the allograft group $(P=0.008$ among the 3 groups, $P=0.011$ for autograft group vs. allograft group, $P=0.007$ for hybrid graft group vs. allograft group), but no statistically significant difference was found between the autograft group and hybrid graft group $(P=0.883)$ (Table 4$)$. 
Table 1 Demographic data of the study groups

\begin{tabular}{lllll}
\hline Variable & Autograft & Hybrid Graft & Allograft & $P$ value \\
\hline Number of patients & 28 & 32 & 37 & $26 / 11$ \\
Gender (male/female), $n$ & $23 / 5$ & $20 / 12$ & $32.8+9.9$ & 0.243 \\
Age, years & $30.1+9.6$ & $29.0+8.4$ & $20 / 17$ & 0.22 \\
Side (left/right), $n$ & $7 / 11$ & $13 / 19$ & $10.4+6.9$ & 0.426 \\
Time from injury to surgery, weeks & $9.4+7.1$ & $10.8+8.1$ & 23 & 8 \\
Meniscal injury & 16 & 16 & 11 & 0.746 \\
Medial meniscus injury, $n$ & 8 & 5 & 4 & 5 \\
Lateral meniscus injury, $n$ & 5 & 6 & 5.59 \\
Medial and Lateral meniscus injury, $n$ & 3 & 5 & 3 & 0.5 \\
MCL injury, $n$ & 5 & $9.0+0.6$ & $19.9+6.6$ \\
Graft diameter, mm & $8.5+0.5$ & $19.4+6.2$ & 0.652 \\
Follow-up, months & $21.1+7.3$ & & 0.000 \\
\hline
\end{tabular}

\section{Discussion}

The most important finding of our study was that the synovial coverage was significantly superior in the hamstring autograft group when compared with hybrid graft and $\gamma$-irradiated allograft groups. In addition, there was no difference between hybrid graft and allograft groups. Concerning the knee laxity evaluated by KT-1000 measurement and subjective graft tension under second-look arthroscopy, both the autograft and hybrid graft groups were significantly superior to those for patients in the allograft group. There were no significant differences in the Lysholm score, IKDC score, or Tegner activity score among the 3 groups during the short-term follow-up.

Hamstring autografts are commonly used for ACL reconstruction, with successful clinical results and low donor site morbidities $[25,26]$. However, the recent literature suggests that a small graft diameter (especially those less than $8 \mathrm{~mm}$ ) would biomechanically decrease the tensile strength [3], and clinically cause high revision risk and poor patient-reported outcomes [5, 6]. Unfortunately, the harvested hamstring tendons showed significant variability in size $[27,28]$, with 7 to $8 \mathrm{~mm}$ being most common for quadruple-stranded grafts [6]. Rather than harvesting additional autograft, surgeons attempt to augment the autograft with allograft tissue to create a hybrid graft. However, only a few studies investigated the clinical outcomes of hybrid ACL reconstructions, with no clear consensus on its effect. Burrus et al. [14] and Wang et al. [16] reported that hybrid hamstring

Table 2 Knee functional assessment at final follow-up at final follow-up

\begin{tabular}{lllll}
\hline Variable & Autograft & Hybrid graft & Allograft & $P$ value \\
\hline Lysholm score & $87.7+8.5$ & $86.8+10.2$ & $85.5+8.0$ & 0.588 \\
IKDC score & $81.9+9.3$ & $82.1+8.9$ & $79.9+8.6$ & 0.544 \\
Tegner activity score & $6.0+1.7$ & $5.9+1.6$ & $5.5+1.6$ & 0.385 \\
\hline
\end{tabular}

ACL grafts led to poorer clinical scores and higher failure rates than autograft hamstring controls. On the contrary, other studies indicated that hybrid graft appeared to be a good treatment option, and hybrid grafts resulted in comparable or even better clinical outcomes and lower failure rates compared with hamstring autograft $[12,13,29,30]$. Based on our findings, we did not find superior effects of ACL reconstruction with hybrid graft than that with autografts, although the graft sizes for the augmented group were significantly larger. The possible reason for this result could be that the irradiated grafts have lower tensile strength compared with nonirradiated or fresh grafts [3, 31, 32]. Therefore, the actually functional tensile strength of hybrid graft might be smaller than the homogeneous hamstrings, although the diameter was larger. This could also explain why the $\gamma$ irradiated allograft ACL reconstruction group could not achieve the same knee stability as homogeneous or augmented hamstrings group in this study.

It has been reported that the ACL grafts undergo a continuous remodeling process of ligamentization consisting of necrosis, revascularization, cellular repopulation, and collagen remodeling [33], and the quality of graft ligamentization is closely related to the grafts' long-term viability [19]. Synovialization plays an important role in graft healing and is considered to affect survival of the graft [34]. Noh et al. [35] and Lee et al. [36] found that the extent of the synovialization is positively correlated with clinical results. The previous studies reported that hamstring autografts showed considerably better synovial coverage than soft tissue allograft based on second-look arthroscopic evaluation [22, 36, 37]. When compared with hybrid graft,

Table 3 Knee stability at final follow-up

\begin{tabular}{lllll}
\hline Variable & Autograft & Hybrid graft & Allograft & $P$ value \\
\hline KT-1000 & $1.6+1.3$ & $1.8+1.3$ & $2.5+1.2$ & 0.009 \\
\hline
\end{tabular}


Table 4 Second-look arthroscopic evaluations of the study groups

\begin{tabular}{llll}
\hline Variable & Autograft (28) & Hybrid graft (32) & Allograft (37) \\
\hline Synovial coverage & 16 & & $P$ value \\
Good & 9 & 8 & 10 \\
Fair & 3 & 16 & 16 \\
Poor & & 8 & 11 \\
Graft tension & 14 & 17 & 7 \\
Taut & 10 & 10 & 19 \\
Mildly lax & 4 & 5 & 11 \\
Lax & & & 0.008 \\
\hline
\end{tabular}

hamstring autograft also showed better extent of synovial coverage after anatomic single-bundle ACL reconstruction [15]. The results of the current study are consistent with those previous studies in that superior synovialization was found with autografts than with allografts or hybrid grafts. Thus, it is reasonable to recommend a homogenous hamstring autograft with effective diameter for primary ACL reconstruction.

One interesting finding of our second-look arthroscopy was that the hybrid group did not show better synovialization than the allograft types, although autograft tissues acted as the main component in hybrid ACL graft. The possible explanation could be that the allograft portion in the hybrid implant was associated with increased inflammation, enhanced immunologic reaction, and slower histologic incorporation, which would slow down the synovialization and the graft remodeling process. Even at approximate 2 years after surgery, the allograft tendons might have inferior graft maturity than autograft tendons in ACL reconstruction [10, 22]. Therefore, we hypothesize that the advantages of increasing the implant size and mechanical properties through allograft augmentation would be compromised by the adverse effect caused by the allografts. Furthermore, the difference in knee laxity between the allograft group and hybrid graft group would diminish over time due to the similar ACL graft synovialization.

The failure rates of hybrid grafts and allografts were reported to be higher than that of autografts after primary ACL reconstruction [2, 14, 16, 38, 39]. However, we did not find significant difference in the graft failure among the three groups. There are several possible factors that led to this result. First, the graft failure was defined as "biological failure" based on arthroscopy in our study [23], which was different from symptomatic "clinical failure" [39] reported in most previous studies. Second, only the patients who underwent second-look arthroscopy were included in this study. The ACL retears based on physical examination, magnetic resonance imaging findings, and side-to-side arthrometer but without arthroscopic examination were not included for analysis. Third, some patient did not return to the full competitive activities at the finial follow-up due to the rehabilitation protocol. We hypothesize that the failure rate would increase in long-term follow-up, because the ACL grafts with poor maturation may not be able to survive intense sports.

\section{Limitation}

There were several limitations in our study. First, the main areas that can be improved on in our future studies include the retrospective design, the limited sample size, and the short-term follow-up. Second, not all the patients who underwent the primary ACL reconstruction were included in our study. We conducted the analysis only on patients who underwent second-look arthroscopy after ACL reconstruction. Therefore, the selection bias could not be avoided. Third, it is quite difficult to evaluate the back of the ACL graft during arthroscopic surgery because it is located deeply in the knee joint. Forth, the assessment on synovial coverage and graft tension depended on the subjective judgment since no standard quantification method has been developed.

\section{Conclusions}

There was no difference in patient-reported knee function among the outcomes of ACL reconstruction with hamstring autografts, hybrid grafts, and allografts in short-term follow-up. However, the hamstring autografts and hybrid grafts produced comparable knee stability and they both outperformed the allografts. The secondlook arthroscopy revealed that hamstring autografts resulted in better synovial coverage than the other two graft types.

\section{Abbreviations}

ACL: Anterior cruciate ligament; IKDC: International Knee Documentation Committee; ROM: Range-of-motion

\section{Acknowledgements}

None. 


\section{Authors' contributions}

$\mathrm{XZ}$ and SG contributed to the study conception and design. YH and PX analyzed and interpreted the data. TL and JG collected the patient samples. $T L, Y F$, and JG prepared the manuscript. All authors read and approved the final manuscript

\section{Funding}

This work has been supported by the project of human resources and social security of Hebei province (No. C20190184).

\section{Availability of data and materials}

The datasets used and/or analyzed during the current study are available from the corresponding author on reasonable request.

\section{Ethics approval and consent to participate}

This study was approved by the ethics committee of the Third Hospital of Hebei Medical University. Written informed consent was obtained from all patients.

\section{Consent for publication}

Not applicable.

\section{Competing interests}

The authors declare that they have no competing interests.

\section{Author details}

'Department of Orthopedics, Third Hospital of Hebei Medical University, No. 139 Ziqiang Road, Shijiazhuang 050051, China. ${ }^{2}$ Key Laboratory of Biomechanics of Hebei Province, No. 139 Ziqiang Road, Shijiazhuang 050051, China. ${ }^{3}$ The Second Department of Intensive Care Unit, Children's Hospital of Hebei Province, No. 133 Jianhua Road, Shijiazhuang 050030, China. ${ }^{4}$ Department of Nuclear medicine, Third Hospital of Hebei Medical University, No. 139 Ziqiang Road, Shijiazhuang 050051, China.

\section{Received: 2 September 2019 Accepted: 30 October 2019}

\section{Published online: 21 November 2019}

\section{References}

1. Shaerf DA, Pastides PS, Sarraf KM, Willis-Owen CA. Anterior cruciate ligament reconstruction best practice: a review of graft choice. World J Orthop. 2014; 5(1):23-9.

2. Tian S, Wang B, Liu L, Wang Y, Ha C, Li Q, et al. Irradiated hamstring tendon allograft versus autograft for anatomic double-bundle anterior cruciate ligament reconstruction: midterm clinical outcomes. Am J Sports Med. 2016; 44(10):2579-88.

3. Boniello MR, Schwingler PM, Bonner JM, Robinson SP, Cotter A, Bonner KF. Impact of hamstring graft diameter on tendon strength: a biomechanical study. Arthroscopy. 2015;31(6):1084-90.

4. Magnussen RA, Lawrence JT, West RL, Toth AP, Taylor DC, Garrett WE. Graft size and patient age are predictors of early revision after anterior cruciate ligament reconstruction with hamstring autograft. Arthroscopy. 2012;28(4):526-31

5. Mariscalco MW, Flanigan DC, Mitchell J, et al. The influence of hamstring autograft size on patient-reported outcomes and risk of revision after anterior cruciate ligament reconstruction: a Multicenter Orthopaedic Outcomes Network (MOON) cohort study. Arthroscopy. 2013;29(12):1948-53.

6. Conte EJ, Hyatt AE, Gatt CJ Jr, Dhawan A. Hamstring autograft size can be predicted and is a potential risk factor for anterior cruciate ligament reconstruction failure. Arthroscopy. 2014;30(7):882-90.

7. Jost PW, Dy CJ, Robertson CM, Kelly AM. Allograft use in anterior cruciate ligament reconstruction. HSS J. 2011;7(3):251-6.

8. Edgar CM, Zimmer S, Kakar S, Jones H, Schepsis AA. Prospective comparison of auto and allograft hamstring tendon constructs for $A C L$ reconstruction. Clin Orthop Relat Res. 2008;466(9):2238-46.

9. Kim YK, Ahn JH, Yoo JD. A comparative study of clinical outcomes and second-look arthroscopic findings between remnant-preserving Tibialis tendon allograft and hamstring tendon autograft in anterior cruciate ligament reconstruction: matched-pair design. Clin Orthop Surg. 2017; 9(4):424-31.
10. Li H, Tao H, Cho S, Chen S, Yao Z, Chen S. Difference in graft maturity of the reconstructed anterior cruciate ligament 2 years postoperatively: a comparison between autografts and allografts in young men using clinical and 3.0-T magnetic resonance imaging evaluation. Am J Sports Med. 2012; 40(7):1519-26.

11. Kaeding CC, Aros B, Pedroza A, Pifel E, Amendola A, Andrish JT, et al. Allograft versus autograft anterior cruciate ligament reconstruction: predictors of failure from a MOON prospective longitudinal cohort. Sports Health. 2011;3(1):73-81.

12. Leo BM, Krill M, Barksdale L, Alvarez-Pinzon AM. Failure rate and clinical outcomes of anterior cruciate ligament reconstruction using autograft hamstring versus a hybrid graft. Arthroscopy. 2016;32(11):2357-63.

13. Jacobs CA, Burnham JM, Makhni E, Malempati CS, Swart E, Johnson DL. Allograft augmentation of hamstring autograft for younger patients undergoing anterior cruciate ligament reconstruction. Am J Sports Med. 2017:45(4):892-9.

14. Burrus MT, Werner BC, Crow AJ, Brockmeier SF, Carson EW, Miller MD, et al. Increased failure rates after anterior cruciate ligament reconstruction with soft-tissue autograft-allograft hybrid grafts. Arthroscopy. 2015;31(12):2342-51.

15. Xu H, Dong J, Xin D, Zhang J, Kang K, Gao S. Second-look arthroscopic evaluation and clinical outcomes of anatomic anterior cruciate ligament reconstruction with autograft and hybrid graft: a retrospective study. Med Sci Monit. 2017;23:5564-73.

16. Wang HD, Gao SJ, Zhang YZ. Comparison of clinical outcomes after anterior cruciate ligament reconstruction using a hybrid graft versus a hamstring autograft. Arthroscopy. 2018;34(5):1508-16.

17. Jackson DW, Corsetti J, Simon TM. Biologic incorporation of allograft anterior cruciate ligament replacements. Clin Orthop Relat Res. 1996; 324:126-33.

18. Cvetanovich GL, Mascarenhas R, Saccomanno MF, Verma NN, Cole BJ, Bush-Joseph $\mathrm{CA}$, et al. Hamstring autograft versus soft-tissue allograft in anterior cruciate ligament reconstruction: a systematic review and meta-analysis of randomized controlled trials. Arthroscopy. 2014;30(12):1616-24.

19. Bottoni CR, Smith EL, Shaha J, Shaha SS, Raybin SG, Tokish JM, et al. Autograft versus allograft anterior cruciate ligament reconstruction: a prospective, randomized clinical study with a minimum 10-year follow-up. Am J Sports Med. 2015;43(10):2501-9.

20. Kondo E, Yasuda K. Second-look arthroscopic evaluations of anatomic double-bundle anterior cruciate ligament reconstruction: relation with postoperative knee stability. Arthroscopy. 2007;23(11):1198-209.

21. Ahn JH, Yoo JC, Yang HS, Kim JH, Wang JH. Second-look arthroscopic findings of 208 patients after ACL reconstruction. Knee Surg Sports Traumatol Arthrosc. 2007;15(3):242-8.

22. Kim SG, Kim SH, Kim JG, Jang KM, Lim HC, Bae JH. Hamstring autograft maturation is superior to tibialis allograft following anatomic single-bundle anterior cruciate ligament reconstruction. Knee Surg Sports Traumatol Arthrosc. 2018;26(4):1281-7.

23. Ménétrey J, Duthon VB, Laumonier T, Fritschy D. "Biological failure" of the anterior cruciate ligament graft. Knee Surg Sports Traumatol Arthrosc. 2008; 16(3):224-31.

24. Kinugasa K, Mae T, Matsumoto N, Nakagawa S, Yoneda M, Shino K. Effect of patient age on morphology of anterior cruciate ligament grafts at second-look arthroscopy. Arthroscopy. 2011;27(1):38-45.

25. Toritsuka Y, Shino K, Horibe S, et al. Second-look arthroscopy of anterior cruciate ligament grafts with multistranded hamstring tendons. Arthroscopy. 2004;20(3):287-93.

26. Goradia VK, Grana WA. A comparison of outcomes at 2 to 6 years after acute and chronic anterior cruciate ligament reconstructions using hamstring tendon grafts. Arthroscopy. 2001;17(4):383-92.

27. Bickel BA, Fowler TT, Mowbray JG, Adler B, Klingele K, Phillips G Preoperative magnetic resonance imaging cross-sectional area for the measurement of hamstring autograft diameter for reconstruction of the adolescent anterior cruciate ligament. Arthroscopy. 2008;24(12):1336-41.

28. Park SY, Oh H, Park S, Lee JH, Lee SH, Yoon KH. Factors predicting hamstring tendon autograft diameters and resulting failure rates after anterior cruciate ligament reconstruction. Knee Surg Sports Traumatol Arthrosc. 2013;21(5):1111-8.

29. Kraeutler MJ, Kim SH, Brown CC, Houck DA, Domby BC, Reynolds KA, et al. Clinical outcomes following primary anterior cruciate ligament reconstruction with hamstring autograft versus planned hybrid graft. J Knee Surg. 2018;31(9):827-33. 
30. Li J, Wang J, Li Y, Shao D, You X, Shen Y. A prospective randomized study of anterior cruciate ligament reconstruction with autograft, $Y$-irradiated allograft, and hybrid graft. Arthroscopy. 2015;31(7):1296-302.

31. Zheng $X, X u$ W, Gu J, Hu Y, Cui M, Feng YE, et al. Effects of graft preconditioning on $\gamma$-irradiated deep frozen tendon allografts used in anterior cruciate ligament reconstruction. Exp Ther Med. 2018;16(2):1338-42.

32. Hamner DL, Brown CH Jr, Steiner ME, Hecker AT, Hayes WC. Hamstring tendon grafts for reconstruction of the anterior cruciate ligament: biomechanical evaluation of the use of multiple strands and tensioning techniques. J Bone Joint Surg Am. 1999;81(4):549-57.

33. Scheffler SU, Unterhauser FN, Weiler A. Graft remodeling and ligamentization after cruciate ligament reconstruction. Knee Surg Sports Traumatol Arthrosc. 2008;16(9):834-42.

34. Lee BI, Min KD, Choi HS, Kwon SW, Chun DI, Yun ES, et al. Immunohistochemical study of mechanoreceptors in the tibial remnant of the ruptured anterior cruciate ligament in human knees. Knee Surg Sports Traumatol Arthrosc. 2009;17(9):1095-101.

35. Noh JH, Yang BG, Roh YH, Lee JS. Synovialization on second-look arthroscopy after anterior cruciate ligament reconstruction using Achilles allograft in active young men. Knee Surg Sports Traumatol Arthrosc. 2011; 19(11):1843-50.

36. Lee JH, Bae DK, Song SJ, Cho SM, Yoon KH. Comparison of clinical results and second-look arthroscopy findings after arthroscopic anterior cruciate ligament reconstruction using 3 different types of grafts. Arthroscopy. 2010; 26(1):41-9.

37. Yoo SH, Song EK, Shin YR, Kim SK, Seon JK. Comparison of clinical outcomes and second-look arthroscopic findings after ACL reconstruction using a hamstring autograft or a tibialis allograft. Knee Surg Sports Traumatol Arthrosc. 2017;25(4):1290-7.

38. Pennock AT, Ho B, Parvanta K, Edmonds EW, Chambers HG, Roocroft JH, et al. Does allograft augmentation of small-diameter hamstring autograft ACL grafts reduce the incidence of graft retear? Am J Sports Med. 2017; 45(2):334-8.

39. Zeng C, Gao SG, Li H, Yang T, Luo W, Li YS, et al. Autograft versus allograft in anterior cruciate ligament reconstruction: a meta-analysis of randomized controlled trials and systematic review of overlapping systematic reviews. Arthroscopy. 2016;32(1):153-63 e18

\section{Publisher's Note}

Springer Nature remains neutral with regard to jurisdictional claims in published maps and institutional affiliations.

Ready to submit your research? Choose BMC and benefit from:

- fast, convenient online submission

- thorough peer review by experienced researchers in your field

- rapid publication on acceptance

- support for research data, including large and complex data types

- gold Open Access which fosters wider collaboration and increased citations

- maximum visibility for your research: over $100 \mathrm{M}$ website views per year

At $\mathrm{BMC}$, research is always in progress.

Learn more biomedcentral.com/submissions 